\title{
La profession de médecin: une profession libérale
}

\section{Jürg Schlup}

Dr, Président de la FMH

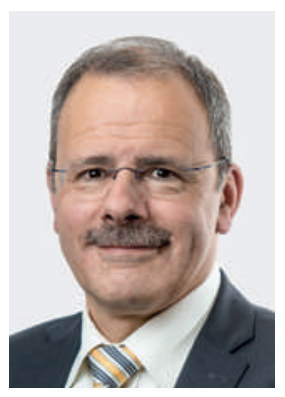

La volonté d'agir «en faveur d'un système de santé efficace et centré sur le patient» se décline dans les Statuts de la FMH en plusieurs sous-objectifs, dont notamment celui de «s'engager en faveur de la liberté et de l'indépendance de la profession médicale». Mais que recouvre cette notion d'exercice libéral de la médecine? L'exercice libéral ne se limite pas à une simple activité indépendante ou à des concepts tels que liberté de s'installer ou liberté thérapeutique. D'ailleurs, seul un tiers environ des personnes qui exercent une profession libérale sont indépendants [1]. Le concept est donc beaucoup plus large.

La désignation de "profession libérale» trouve son origine dans les sept arts libéraux, les colonnes de la sagesse enseignées dans l'Antiquité, regroupant les disciplines "dignes de l'homme libre», dont l'objectif premier n'est pas la subsistance [2]. Au Moyen Age cependant, cette approche a évolué. Les études d'«artes liberales» ouvraient la voie à une profession technique, médicale ou juridique et à une activité au service de la communauté, créant un lien entre citoyen et Etat [2]. L'exercice «libre/libéral» de tâches «souveraines» conférait des responsabilités élevées et légitimait l'octroi de quelques privilèges [2].

\section{Confiance et responsabilité sont au cœur de la notion de profession libérale.}

Aujourd'hui, les professions libérales ont quatre caractéristiques principales, que le Conseil fédéral décrit pour la Suisse de la façon suivante [1]: Exercer une profession libérale consiste à fournir des services - souvent à composante intellectuelle, mais surtout reliés à la personne: «Toute personne qui exerce une profession libérale le fait à titre personnel et sous sa propre responsabilité. Elle ne peut être remplacée facilement, car elle fait partie intégrante de la prestation de service», qui implique surtout une relation de confiance. L'exercice d'une profession libérale suppose une qualification professionnelle élevée, une forte autonomie ainsi que des caractéristiques supplémentaires, comme d'avoir excellente réputation et de répondre à des «exigences élevées d'ordre éthique». Enfin, pour satisfaire aux trois socles - liberté, responsabilité et confiance -, chaque profession libérale établit des règles professionnelles qui vont au-delà de la qualification, et assume ainsi une double responsabilité: édicter des règles et les faire respecter [2].

A l'intérieur de ce groupe très hétérogène de professions libérales, ces critères s'appliquent particulièrement bien à l'exercice de la médecine. En effet, la confiance que le patient accorde à son médecin, aussi bien pour son humanité que pour son expertise, mais aussi les aspects éthiques liés à la pratique, et mis en exergue dans notre Code de déontologie, et la responsabilité que le médecin assume pour la santé de ses patients font de la liberté un élément essentiel de notre travail.

\section{La liberté est indissociable de l'exercice de la profession de médecin.}

Que les jeunes médecins soient aujourd'hui le plus souvent salariés n'est aucunement en contradiction avec le fait d'exercer librement la médecine - cela implique une certaine vigilance face aux limites posées par le cadre professionnel. En revanche, d'autres évolutions ont un impact beaucoup plus important sur la liberté d'exercer. Je pense notamment aux conflits entre pression économique et conscience professionnelle, à la substitution exigée sous couvert d'interprofessionnalité, aux atteintes portées au secret médical, à l'intervention tarifaire du Conseil fédéral et à la menace d'une surrégulation de notre profession. Ces défis devraient aiguiser notre conscience quant à la signification que nous donnons à un exercice libéral. En parallèle, notre compréhension de la responsabilité qui en découle doit pouvoir évoluer avec le temps pour que nous puissions la vivre et la transmettre.

\section{Références}

1 Rapport du Conseil fédéral «Professions libérales: quel est leur poids dans l'économie nationale?», 15.1.2014.

2 Gentinetta K. Zur Freiheit berufen. Dans la publication 20 ans d'USPL; 2010. 